\title{
MELHORA DA ESTABILIDADE DINÂMICA DE SISTEMAS ELÉTRICOS DE POTÊNCIA MULTIMÁQUINAS USANDO O DISPOSITIVO FACTS "THYRISTOR-CONTROLLED SERIES CAPACITOR - TCSC"
}

\author{
Marcos Amorielle Furini* \\ mafuriniealuno.feis.unesp.br
}

\author{
Percival Bueno de Araujo* \\ percivaledee.feis.unesp.br
}

* Departamento de Engenharia Elétrica

Faculdade de Engenharia de Ilha Solteira - FEIS/UNESP

Av. Brasil Norte, 364, 15385-000 Ilha Solteira, SP, Brasil

\begin{abstract}
RESUMO
O principal objetivo deste trabalho é analisar o desempenho dinâmico de um sistema elétrico de potência multimáquinas com a consideração do dispositivo FACTS TCSC (do inglês, "Thyristor Controlled Series Capacitor"). Para tanto será utilizado o Modelo de Sensibilidade de Potência para o sistema elétrico e será definido um modelo dinâmico para o TCSC. Através de simulações de um sistema teste serão obtidas informações a respeito da estabilidade do sistema elétrico de potência frente a pequenas perturbações.
\end{abstract}

PALAVRAS-CHAVE: Estabilidade de Sistemas de Potência, Estabilidade a Pequenas Perturbações, FACTS, TCSC.

\footnotetext{
ABSTRACT subjected to small disturbances.

Artigo submetido em 28/05/2007

1a. Revisão em $07 / 08 / 2007$

2a. Revisão em 22/12/2007

3a. Revisão em 27/02/2008

Aceito sob recomendação do Editor Associado Prof. Carlos A. Castro
}

The main objective of this work is to analyze the dynamic performance of a multimachine power system with consideration of FACTS device TCSC ("Thyristor-Controlled Series Capacitor"). The Power Sensitivity Model for electric systems will be used and will be defined a dynamic model for the TCSC. Simulation tests were effectuated to obtain information about the stability of the electric power system when
KEYWORDS: Power System Stability, Small-signal Stability, FACTS, TCSC.

\section{INTRODUÇÃO}

Os avanços tecnológicos da humanidade solicitam continuamente aumentos na demanda de energia elétrica. Portanto, caracteriza-se como principal função dos sistemas elétricos de potência o suprimento dessa demanda crescente de energia elétrica de forma segura, confiável e economicamente viável. Para aumentar a confiabilidade a interligação de sistemas elétricos de potência é cada vez maior, originando assim interações de diversos tipos de equipamentos com comportamentos dinâmicos diferentes.

O estudo do comportamento de um sistema elétrico, quando este é submetido a perturbações, é reconhecido como o estudo da estabilidade do sistema elétrico. Perturbações de pequena escala em forma de desvios de carga ocorrem continuamente, ocasionando ajustes na geração de energia e caracterizam o estudo da estabilidade dinâmica (ou estabilidade a pequenas perturbações). Uma perturbação é considerada pequena se as equações que descrevem o comportamento do sistema podem ser linearizadas em torno de um ponto de equilíbrio estável e assim, as técnicas de análise linear podem ser adotadas (Kundur, 1994).

As oscilações eletromecânicas de baixa freqüência são comumente relacionadas às interações das oscilações do ângulo do rotor das máquinas geradoras e caracterizam o principal 
motivo de instabilidade provocada por pequenas perturbações. Tais oscilações são preponderantes em grandes sistemas interligados, onde o transporte de energia é realizado por longas linhas de transmissão com altas reatâncias indutivas. Para o estudo de seus efeitos no comportamento dinâmico do sistema elétrico necessita-se de uma detalhada modelagem. Neste trabalho será utilizado o modelo linear proposto em (Deckmann e da Costa, 1994), intitulado Modelo de Sensibilidade de Potência.

A realização de compensação de reativos tem sido largamente utilizada para aumentar a capacidade de transmissão e, mais recentemente, tem sido utilizada para o amortecimento de oscilações eletromecânicas. No passado, esta compensação era realizada pela inserção manual de um banco de capacitores em série com a linha de transmissão. O resultado desta operação era a diminuição da reatância efetiva da linha de transmissão, tornando o sistema de potência eletricamente mais robusto. Porém, com o avanço tecnológico na área de eletrônica de potência, dispositivos automáticos de compensação foram desenvolvidos, os quais receberam o nome de Controladores FACTS ("Flexible Alternating Current Transmission Systems") (Song e Johns, 1999).

Dentre os diversos dispositivos FACTS existentes está o Capacitor Série Controlado a Tiristor (TCSC), o qual será utilizado neste trabalho.

Para a análise do comportamento dinâmico do sistema elétrico, voltada para as oscilações eletromecânicas, propõe-se a inclusão do controlador FACTS TCSC, descrito por um modelo dinâmico, cujo sinal de entrada para seu controle é o fluxo de potência ativa na linha compensada. São realizados estudos sobre o amortecimento inserido no sistema devido às diferentes localizações do TCSC no sistema elétrico.

\section{MODELO DE SENSIBILIDADE DE PO- TÊNCIA}

O modelo de sensibilidade de potência descrito em Deckmann e da Costa (1994) é uma ferramenta de análise linear do comportamento dinâmico de sistemas elétricos de potência. Esta modelagem possui uma importante e positiva característica quando comparado com o modelo de Heffron \& Phillips (DeMello e Concordia, 1969), amplamente utilizado na literatura para o estudo da estabilidade a pequenas perturbações. Esta característica é a preservação de todas as barras do sistema elétrico no modelo, não ocorrendo a redução às barras internas dos geradores. Como conseqüência, há a manutenção da integridade do sistema elétrico de potência.

A dedução do modelo de sensibilidade de potência é baseada no conceito fundamental da aplicação do balanço de potência elétrica, ativa e reativa, em cada barra do sistema durante

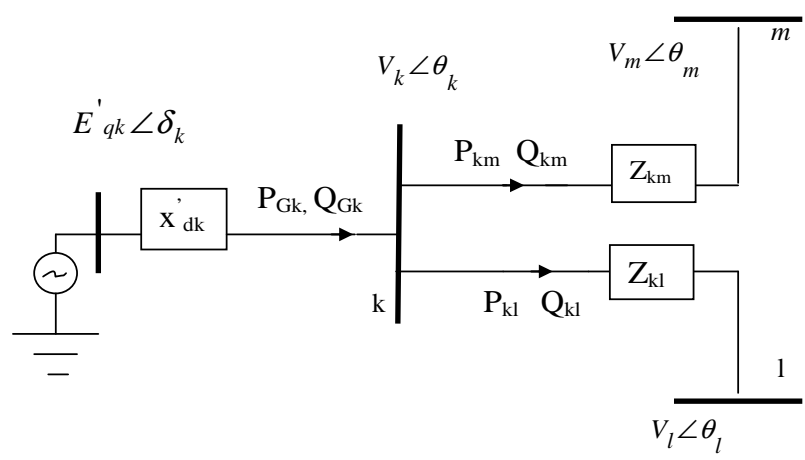

Figura 1: Sistema Elétrico de Potência

todo e qualquer processo dinâmico. Esta dedução pode ser realizada utilizando-se um diagrama genérico do sistema elétrico, como mostrado na Figura 1.

Este diagrama mostra uma barra genérica $k$ com tensão $V_{k}$, que possui um gerador síncrono representado por sua tensão interna $E_{k}^{\prime}$ e reatância transitória de eixo direto $x_{d k}^{\prime}$. Este gerador está conectado a um conjunto $\Omega_{k}$ de barras do sistema elétrico, através de linhas de transmissão representadas por impedâncias $Z_{k m}=R_{k m}+j X_{k m}$.

\subsection{Equações Gerais do Modelo}

A aplicação do balanço de potência ativa e reativa na barra genérica $k$ pode ser descrita por (1) e (2), respectivamente.

$$
\begin{gathered}
P_{G k}-\sum_{m \in \Omega_{k}} P_{k m}=0 \\
Q_{G k}-\sum_{m \in \Omega_{k}} Q_{k m}=0
\end{gathered}
$$

As potências ativa e reativa fornecidas pelos geradores podem ser obtidas através do cálculo da potência entregue à barra $k$ e são descritas em (3) e (4), mostradas na seqüência.

$$
\begin{gathered}
P_{G k}=\frac{E_{q k}^{\prime} V_{k} \operatorname{sen}\left(\delta_{k}-\theta_{k}\right)}{x_{d k}^{\prime}}+ \\
\frac{1}{2}\left[\frac{1}{x_{q k}}-\frac{1}{x_{d k}^{\prime}}\right] V_{k}^{2} \operatorname{sen} 2\left(\delta_{k}-\theta_{k}\right) \\
Q_{G k}=\frac{E_{q k}^{\prime} V_{k} \cos \left(\delta_{k}-\theta_{k}\right)}{x_{d k}^{\prime}}- \\
\frac{V_{k}^{2}}{x_{d k}^{\prime}}-\frac{V_{k}^{2}}{2}\left[\frac{1}{x_{q k}}-\frac{1}{x_{d k}^{\prime}}\right] \cdot\left[1-\cos 2\left(\delta_{k}-\theta_{k}\right)\right]
\end{gathered}
$$


Os fluxos de potência ativa e reativa nas linhas de transmissão podem ser obtidos de maneira semelhante e são descritos por (5) e (6), respectivamente.

$$
\begin{gathered}
P_{k m}=\frac{R_{k m}}{\left|Z_{k m}\right|^{2}}\left[V_{k}^{2}-V_{k} V_{m} \cos \left(\theta_{k}-\theta_{m}\right)\right]+ \\
\frac{X_{k m}}{\left|Z_{k m}\right|^{2}} V_{k} V_{m} \operatorname{sen}\left(\theta_{k}-\theta_{m}\right) \\
Q_{k m}=-\frac{R_{k m}}{\left|Z_{k m}\right|^{2}}\left[V_{k} V_{m} \sin \left(\theta_{k}-\theta_{m}\right)\right]+ \\
\frac{X_{k m}}{\left|Z_{k m}\right|^{2}}\left[V_{k}^{2}-V_{k} V_{m} \cos \left(\theta_{k}-\theta_{m}\right)\right.
\end{gathered}
$$

\subsection{Equações Dinâmicas do Modelo}

O comportamento dinâmico das grandezas mecânicas da máquina síncrona (a saber, velocidade angular - $\omega$, e ângulo interno - $\delta$ ), pode ser descrito a partir da equação de oscilação ("swing") do gerador, a qual se decompõe em duas equações diferenciais de primeira ordem (Kundur, 1994; Anderson e Fouad, 2003).

Estas equações são mostradas em (7) e (8), onde $M_{k}$ é a constante de inércia do gerador síncrono, $D_{k}$ o coeficiente de amortecimento introduzido no sistema elétrico devido à presença das diversas cargas e $\omega_{0}$ é a velocidade síncrona.

$$
\begin{gathered}
\dot{\omega}_{k}=\frac{1}{M_{k}}\left(P_{m k}-P_{G k}-D_{k} \omega_{k}\right) \\
\dot{\delta_{k}}=\omega_{0} \omega_{k}
\end{gathered}
$$

O comportamento dinâmico da tensão interna da máquina síncrona ( $\left.E_{q k}^{\prime}\right)$ é descrito por (9) (Kundur, 1994; Anderson e Fouad, 2003).

$$
T_{d 0 k}^{\prime} \dot{E}_{q k}^{\prime}=E_{f d k}-\left[E_{q k}^{\prime}+\left(x_{d k}-x_{d k}^{\prime}\right) I_{d k}\right]
$$

Para a consideração dos efeitos da variação da tensão de excitação $\left(E_{f d}\right)$ da máquina síncrona se faz necessário introduzir o Regular Automático de Tensão. Este dispositivo possui a função de regular a tensão de excitação de acordo com as variações da tensão terminal em relação a uma tensão de referência especificada. Dentre os diversos modelos do sistema de excitação encontrados na literatura, neste trabalho

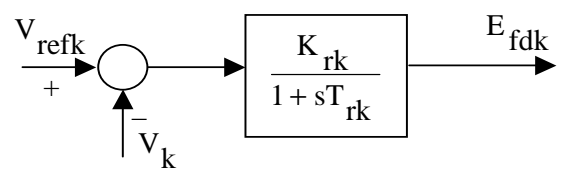

Figura 2: Regulador Automático de Tensão

será considerado um modelo de primeira ordem, de acordo com o diagrama mostrado na Figura 2.

Baseando-se no diagrama da Figura 2 tem-se o comportamento dinâmico da tensão de campo do gerador, descrito matematicamente em (10).

$$
\underset{f d k}{\stackrel{\bullet}{E}}=-\frac{1}{T_{r k}} E_{f d k}+\frac{K_{r k}}{T_{r k}} V_{r e f k}-\frac{K_{r k}}{T_{r k}} V_{k}
$$

\subsection{Linearização}

Neste trabalho a análise do comportamento dinâmico do sistema elétrico de potência será focada na estabilidade dinâmica, ou seja, a estabilidade a pequenas perturbações. Sendo assim, as equações 1-10 (de maneira geral, não lineares), serão linearizadas em torno de um ponto de equilíbrio estável para a obtenção do modelo de sensibilidade de potência. As equações resultantes são mostradas nas expressões 11 a 20 (As expressões para os coeficientes encontram-se no Apêndice $\mathrm{A})$.

- Potências fornecidas:

$\Delta P_{G k}=A 1_{G k} \Delta\left(\delta_{k}-\theta_{k}\right)+A 2_{G k} \Delta E_{q k}^{\prime}+A 3_{G k} \Delta V_{k}$

$\Delta Q_{G k}=R 1_{G k} \Delta\left(\delta_{k}-\theta_{k}\right)+R 2_{G k} \Delta E_{q k}^{\prime}+R 3_{G k} \Delta V_{k}$

- Fluxos de potência nas linhas de transmissão:

$$
\Delta P_{k m}=A 1_{k m} \Delta \theta_{k m}+A 2_{k m} \Delta V_{k}+A 3_{k m} \Delta V_{m}
$$

$$
\Delta Q_{k m}=R 1_{k m} \Delta \theta_{k m}+R 2_{k m} \Delta V_{k}+R 3_{k m} \Delta V_{m}
$$

- Equações de oscilação da máquina síncrona:

$$
\Delta \dot{\omega}_{k}=\frac{1}{M_{k}}\left(\Delta P_{m k}-\Delta P_{G k}-D_{k} \Delta \omega_{k}\right)
$$




$$
\Delta \dot{\delta_{k}}=\omega_{0}\left(\Delta \omega_{k}\right)
$$

- Equação da tensão interna da máquina síncrona

$$
\begin{aligned}
T_{d 0 k}^{\prime} \Delta \dot{E}_{q k}^{\prime}=\Delta E_{f d k}-\frac{x_{d k}}{x_{d k}^{\prime}} \Delta E_{q k}^{\prime}+ \\
K_{V k} \Delta V_{k}-K_{A k} \Delta\left(\delta_{k}-\theta_{k}\right)
\end{aligned}
$$

Os parâmetros $K_{V}$ e $K_{A}$ são chamados de "coeficientes da reação de armadura" e são dados por (18) e (19), respectivamente. A reação de armadura representa a relutância da máquina síncrona a perturbações externas.

$$
\begin{gathered}
K_{V k}=\frac{\left(x_{d k}-x_{d k}^{\prime}\right) \cos \left(\delta_{k}-\theta_{k}\right)}{x_{d k}^{\prime}} \\
K_{A k}=\frac{\left(x_{d k}-x_{d k}^{\prime}\right) \cdot V_{k} \operatorname{sen}\left(\delta_{k}-\theta_{k}\right)}{x_{d k}^{\prime}}
\end{gathered}
$$

- Equação de campo (Regulador Automático de Tensão)

$$
\Delta \underset{f d k}{\stackrel{\bullet}{E}}=-\frac{1}{T_{r k}} \Delta E_{f d k}+\frac{K_{r k}}{T_{r k}} \Delta V_{r e f k}-\frac{K_{r k}}{T_{r k}} \Delta V_{k}
$$

\subsection{Representações do Sistema Elétrico}

A utilização do sistema de equações dadas por (11) a (20) permite representar o modelo de sensibilidade de potência do sistema elétrico de potência multimáquinas nos domínios do tempo e da frequiência. Nestas representações ocorrem variáveis de estado e variáveis algébricas.

Para a definição destas representações considere um sistema multimáquinas de $n g$ geradores e $n b$ barras e os vetores dados por (21), (22) e (23).

$$
\begin{aligned}
\Delta x= & {\left[\left[\Delta \omega_{1} \cdots \Delta \omega_{n g}\right]\left[\Delta \delta_{1} \cdots \Delta \delta_{n g}\right]\right.} \\
& {\left.\left[\Delta E_{q 1}^{\prime} \cdots \Delta E_{q_{n g}}^{\prime}\right]\left[\Delta E_{f d 1} \cdots \Delta E_{f d_{n g}}\right]\right]^{t} }
\end{aligned}
$$

$$
\Delta u=\left[\begin{array}{ll}
{\left[\Delta P_{m 1} \cdots \Delta P_{m_{n g}}\right]} & {\left[\Delta V_{r e f 1} \cdots \Delta V_{r e f}\right]}
\end{array}\right]^{t}
$$

$$
\Delta z=\left[\begin{array}{lll}
{\left[\Delta \theta_{1} \cdots \Delta \theta_{n b}\right]} & {\left[\Delta V_{1} \cdots \Delta V_{n b}\right]}
\end{array}\right]^{t}
$$

Desta forma, o comportamento dinâmico do sistema elétrico pode ser representado no domínio do tempo conforme (24), onde são considerados os vetores de variáveis de estado $(\Delta x)$, de entradas $(\Delta u)$, e variáveis algébricas $(\Delta z)$.

$$
\left[\begin{array}{c}
\Delta \dot{x} \\
0
\end{array}\right]=\left[\begin{array}{ll}
J_{11} & J_{12} \\
J_{21} & J_{22}
\end{array}\right]\left[\begin{array}{c}
\Delta x \\
\Delta z
\end{array}\right]+\left[\begin{array}{l}
B_{1} \\
B_{2}
\end{array}\right] \Delta u
$$

A representação do sistema elétrico de potência no domínio da frequiência é obtida através da aplicação da transformada de Laplace no sistema de equações (11-20), cujo diagrama de blocos é mostrado na Figura 3, onde para facilidade de notação, é representado apenas um gerador genérico $k$.

\section{CONTROLADOR FACTS TCSC}

Com o aumento crescente da demanda de energia elétrica, os sistemas de potência tornaram-se mais complexos em dimensão e utilização de aparelhos, fato que aliado às pressões de mercado exigiu que tais sistemas operassem próximos de seus limites térmicos e de estabilidade (Paserba, 2003). A

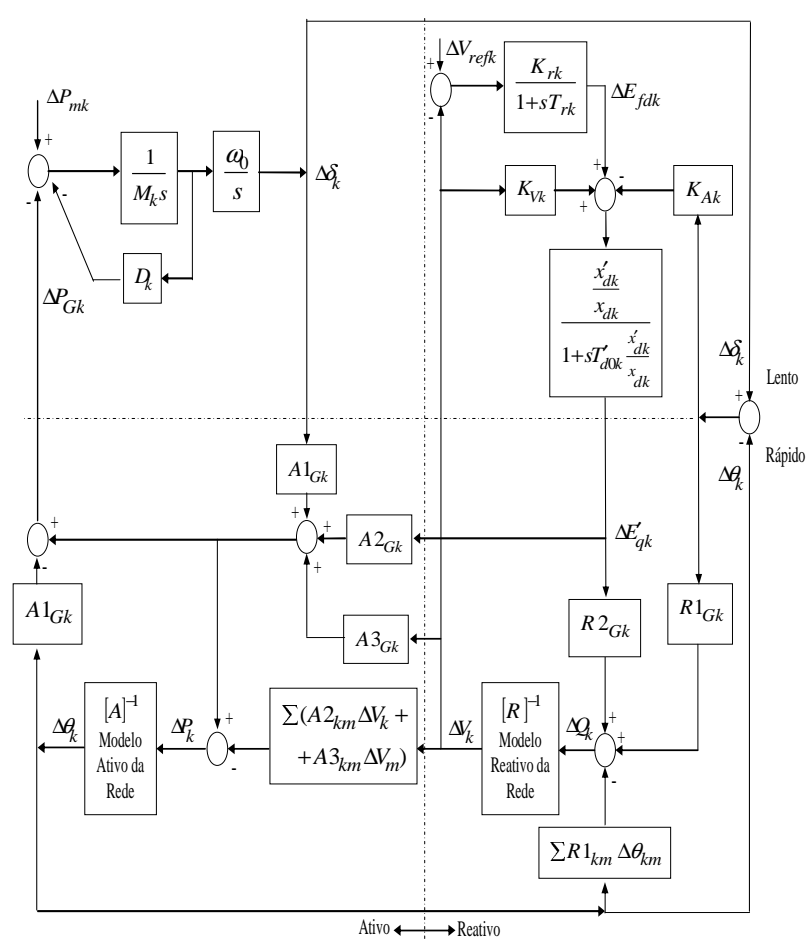

Figura 3: Diagrama de Blocos do Sistema Multimáquinas 
construção de novos sistemas de transmissão pode não ser a solução que represente menor tempo e custos de implementação, por razões ambientais e econômicas. Para tentar sanar as dificuldades (ou mesmo impossibilidade) de transmissão de energia elétrica surgem aplicações de dispositivos que aumentam a eficácia do sistema de transmissão, os chamados controladores FACTS.

A ação destes controladores se baseia no fato de que o fluxo de potência elétrica ativa em uma linha de transmissão, desconsiderando-se as perdas, é obtido da relação entre os módulos das tensões da barra inicial e final, o seno da diferença angular entre essas barras e a reatância da linha, de acordo com (25) (Song e Johns, 1999).

$$
P_{i f}=\frac{V_{i} V_{f} \sin \left(\theta_{i}-\theta_{f}\right)}{X_{i f}}
$$

A ação dos controladores FACTS é atuar sobre os parâmetros de (25) de maneira a maximizar o fluxo de potência na linha de transmissão. Basicamente, os controladores podem ser divididos em série ou shunt, sendo os primeiros responsáveis pela compensação da reatância da linha de transmissão, enquanto os segundos realizam a regulação da tensão terminal da barra em que se encontra instalado.

Neste trabalho são estudados os benefícios proporcionados pela inclusão do controlador série TCSC no estudo da estabilidade a pequenas perturbações.

\subsection{Modelo Dinâmico do TCSC}

O TCSC, como o próprio nome diz, é um dispositivo dotado de uma capacitância fixa e um reator controlado a tiristores. A reatância efetiva do TCSC é modificada através do controle do disparo dos tiristores e assim inserida na linha de transmissão, de maneira a manipular a reatância total da linha e, conseqüentemente, controlar seu fluxo de potência. Um esquema básico para o TCSC é mostrado na Figura 4 (Song e Johns, 1999).

Para estudos de estabilidade a pequenas perturbações o

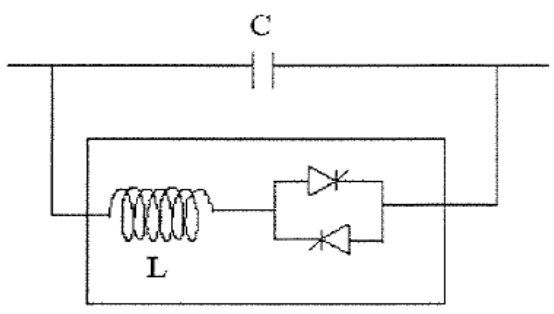

Figura 4: Esquema Básico do TCSC
TCSC pode ser modelado matematicamente como uma reatância variável $\left(X_{T C S C}\right)$, que provoca a diminuição da reatância original da linha $\left(X_{k m}\right)$, resultando em uma reatância efetiva $X_{k m e f}$, como mostra (26).

$$
X_{k m e f}=X_{k m}-X_{T C S C}
$$

Um modelo dinâmico linearizado para o TCSC é apresentado na Figura 5, onde o sinal de controle utilizado é a potência ativa na linha de transmissão, conforme sugerido por Domingues et al. (2002).

O uso do fluxo de potência ativa da linha de transmissão onde o TCSC encontra-se instalado enquadra-se na filosofia de utilização de sinais locais, o que dispensa o uso de sistemas de telecomunicação, os quais oneram economicamente e diminuem a confiabilidade da operação. Outro fator positivo é que a modulação da reatância do TCSC tem sua eficiência aumentada com o aumento do fluxo interárea o que, conseqüentemente, introduzirá maior amortecimento para condições de alto carregamento (Pellanda, et al., 2006). Este sinal é processado por um controlador que fornece amortecimento ao sistema, comumente denominado POD ("Power Oscillation Damping"). De acordo com a Figura 5, esse controlador é composto por um ganho estático $\mathrm{K}_{P O D}$, que é ajustado de forma a obter o amortecimento desejado; um bloco "washout" definido pela constante de tempo Tw; as constantes de tempo T1 e T3 responsáveis pelo avanço de fase e as constantes de tempo T2 e T4 utilizadas para o fornecimento de atraso de fase ao sinal de entrada. Por fim considerou-se a constante de tempo $T_{T C S C}$, que representa o atraso inerente ao sistema de controle (Kundur, 1994).

A partir do diagrama mostrado na Figura 5 é obtida a equação linearizada que descreve o comportamento dinâmico do TCSC no sistema de energia elétrica, mostrada em (27).

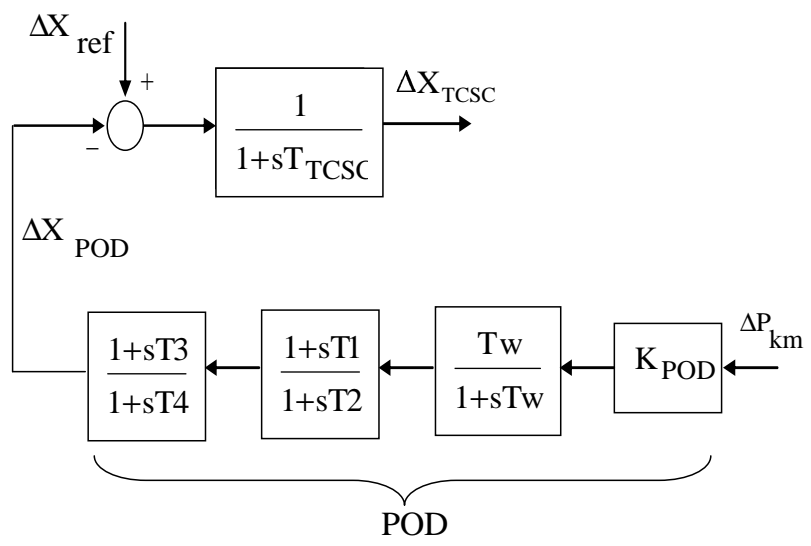

Figura 5: Modelo Dinâmico do TCSC 
$\Delta \underset{T C S C}{\stackrel{\dot{X}}{X}}=\frac{1}{T_{T C S C}}\left(\Delta X_{r e f}-\Delta X_{T C S C}-P O D(s) \Delta P_{k m}\right)$

\subsection{Projeto do Controlador POD do TCSC}

O projeto do controlador POD é baseado na obtenção dos resíduos da função de transferência em malha aberta entre a entrada $\left(\Delta \mathrm{P}_{k m}\right)$ e a saída $\left(\Delta \mathrm{X}_{P O D}\right)$ do controlador POD conforme proposto por Yang et al. (1998) e mostrado em (28). O resíduo $\left(R_{i j k}\right)$ obtido, fornece informações sobre a controlabilidade e observabilidade do conjunto entrada-saída $\left(C_{j}--B_{k}\right)$ para o controlador em um determinado autovalor $\left(\lambda_{i}\right)$ de acordo com os autovetores direito $\left(\phi_{i}\right)$ e esquerdo $\left(\psi_{i}\right)$ obtidos da matriz de estados do sistema. Portanto, o maior resíduo fornecerá maior amortecimento ao modo de oscilação selecionado. É importante notar que este método também pode ser utilizado para determinação da mais efetiva locação do TCSC no sistema.

$$
R_{i j k}=C_{j} \varphi_{i} \psi_{i} B_{k}
$$

Conforme descrito em Yang et al. (1998) pode-se deslocar o autovalor $\left(\lambda_{i}\right)$ para o semiplano esquerdo com a inclusão do controlador, de maneira que sua componente real se torne mais negativa. A equação (29) mostra a relação entre o deslocamento do autovalor e o seu correspondente resíduo.

$$
\Delta \lambda_{i}=R_{i j k} P O D\left(\lambda_{i}\right)=R_{i j k}\left(K_{P O D} H\left(\lambda_{i}\right)\right)
$$

O projeto do controlador se resume a calcular as constantes de tempo $T 1=T 3, T 2=T 4$ e o ganho $K_{P O D}$ de forma a introduzir a compensação de fase necessária ao deslocamento do autovalor de interesse. Esse processo é descrito por Aboul-Ela et al. (1996) para dispositivos PSS ("Power System Stabilizers"), sendo que este mesmo procedimento é aplicável para controladores de dispositivos FACTS. Para isso considere que o ângulo a ser compensado pelo controlador seja $\beta, \omega_{i}$ seja a freqüência em rad/s do modo eletromecânico de interesse e que $\lambda_{\text {ides }}$ a posição desejada para o autovalor, de forma que o conjunto de equações (30) fornece o procedimento para obtenção dos parâmetros do controlador.

$$
\begin{array}{r}
\beta=180^{\circ}-\arg \left(R_{i j k}\right) \\
\alpha=\frac{1-\operatorname{sen}(\beta / 2)}{1+\operatorname{sen}(\beta / 2)} \\
T 2=\frac{1}{\omega_{i} \sqrt{\alpha}} ; \quad T 1=\alpha T 2 \\
K_{P O D}=\left|\frac{\lambda_{i d e s}-\lambda_{i}}{R_{i j k} H\left(\lambda_{i}\right)}\right|
\end{array}
$$

Com o sistema elétrico e o controlador FACTS TCSC devidamente modelados será estudado o comportamento dinâmico de um sistema exemplo, para se avaliar a influência do compensador na estabilidade a pequenas perturbações.

\section{SISTEMA MULTIMÁQUINAS UTILIZADO}

O estudo da estabilidade a pequenas perturbações de sistemas elétricos multimáquinas fornece informações sobre o comportamento dinâmico de cada máquina geradora e também sobre as interações das oscilações eletromecânicas entre essas máquinas, após a ocorrência de uma pequena perturbação em qualquer parte do sistema.

Neste trabalho será utilizado o sistema multimáquinas hipotético de duas áreas e simétrico, proposto por Klein et al. (1991). As áreas possuem dois geradores idênticos e uma carga passiva, e são interligadas por três linhas de transmissão longas em paralelo, conforme mostrado na Figura 6. Os principais dados deste sistema são listados no Apêndice B.

\subsection{Modos Eletromecânicos de Oscilação}

Em grandes sistemas de energia elétrica ocorrem diversos tipos de oscilações como, por exemplo, modos de oscilação devidos aos sistemas de controle da excitação e de velocidade da máquina síncrona. Os principais modos para o estudo da estabilidade a pequenas perturbações são aqueles associados

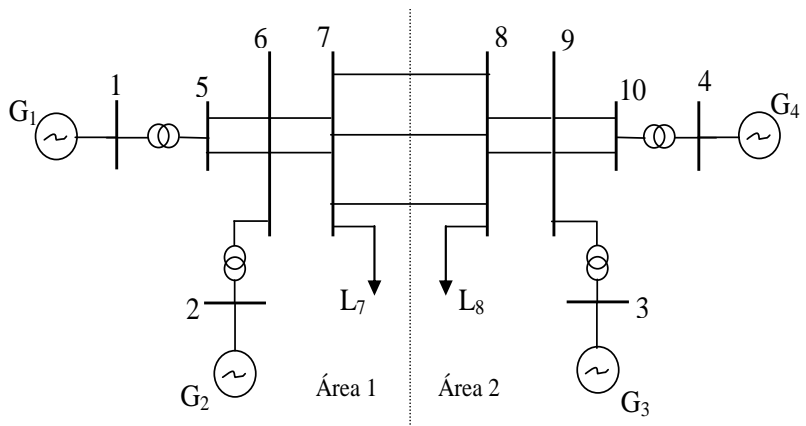

Figura 6: Diagrama Unifilar do Sistema Multimáquinas de Duas Áreas 
às oscilações dos rotores das máquinas síncronas, que são denominados de "modos eletromecânicos de oscilação". Tais modos podem ser classificados de acordo com sua freqüência de oscilação, sendo os de maior interesse os modos locais e os modos interárea. Modos locais de oscilação se encontram na faixa de 0,7 a 2,0 Hz e estão associados às oscilações dos rotores de um grupo de geradores próximos, fisicamente ou eletricamente. Modos interárea de oscilação localizam-se na faixa de 0,1 a $0,8 \mathrm{~Hz}$ e são relacionados com as oscilações de grupos de geradores de uma área contra outro grupo de geradores de outra área (Kundur, 1994; Anderson e Fouad, 2003; Klein et al., 1991).

No sistema multimáquinas considerado nota-se claramente a existência de duas áreas simétricas, sendo estas conectadas por três linhas de transmissão paralelas longas, ou seja, com alta reatância indutiva. Tal fato contribui para a falta de amortecimento do sistema, ocasionando instabilidade. Isto pode ser evidenciado, segundo o primeiro método de Lyapunov, através da análise das raízes da equação característica do sistema, ou seja, pela análise dos autovalores da matriz de estados do sistema, conforme mostrado na Tabela 1.

Tabela 1: Autovalores Dominantes, Freqüência e Coeficiente de Amortecimento $(\zeta)$.

\begin{tabular}{|c|c|c|}
\hline Autovalores & Freqüîncia $(\mathbf{H z})$ & $\zeta$ \\
\hline $\begin{array}{c}-0,2356 \pm \mathrm{j} 6,2956 \\
(\text { Local } 1)\end{array}$ & 1,0026 & 0,0374 \\
\hline $\begin{array}{c}-0,1585 \pm \mathrm{j} 5,8779 \\
(\text { Local } 2)\end{array}$ & 0,9358 & 0,0270 \\
\hline $\begin{array}{c}0,0460 \pm \mathrm{j} 4,1382 \\
\text { (interárea) }\end{array}$ & 0,6587 & $-0,0111$ \\
\hline
\end{tabular}

De acordo com os dados da Tabela 1, nota-se que o autovalor relacionado ao modo de oscilação interárea é o responsável pela instabilidade do sistema. Desta maneira, medidas devem ser tomadas para se tentar estabilizar o sistema de potência. Neste trabalho, a medida considerada é a inclusão do controlador FACTS TCSC.

\section{SIMULAÇÕES E RESULTADOS}

Nesta seção são apresentados os resultados e simulações obtidos com a inclusão do modelo dinâmico do TCSC no sistema elétrico da Figura 6, onde o TCSC é inserido nas linhas de transmissão de maneira a verificar o melhor local para sua instalação, seu efeito sobre os autovalores do sistema devido a diferentes localizações e o comportamento dinâmico do sistema quando submetido a pequenas perturbações.

\subsection{Efeitos Sobre os Autovalores do Sis- tema Elétrico}

Conforme descrito anteriormente, a localização mais efetiva para o TCSC pode ser obtida do cálculo dos resíduos da função de transferência entre a entrada e saída do controlador POD.

A Tabela 2 mostra o módulo dos resíduos correspondentes aos autovalores, associados aos modos eletromecânicos locais e interárea para as possíveis localizações do TCSC no sistema. Pode-se notar que a magnitude dos resíduos depende da localização do TCSC no sistema.

Tabela 2: Módulo dos Resíduos Associados aos Modos Eletromecânicos para diversas Localizações do TCSC.

\begin{tabular}{|c|c|c|c|}
\hline LT & Local 1 & Local 2 & Interárea \\
\hline $1-5$ & 140,230 & 1,4993 & 20,201 \\
\hline $2-6$ & 274,220 & 0,2869 & 21,908 \\
\hline $5-6$ & 142,580 & 1,4028 & 20,018 \\
\hline $6-7$ & 1,3265 & 2,7771 & 166,520 \\
\hline $7-8$ & 0,7349 & 0,3717 & 27,869 \\
\hline $9-8$ & 5,0980 & 1,2624 & 137,990 \\
\hline $3-9$ & 6,1628 & 264,060 & 14,047 \\
\hline $10-9$ & 0,0917 & 154,860 & 18,388 \\
\hline $4-10$ & 0,0889 & 152,980 & 18,687 \\
\hline
\end{tabular}

De acordo com a Tabela 2, a instalação do TCSC na área 1 terá maior influência no modo local 1 , sendo que no caso de instalação na área 2 sobre o modo local 2. Nota-se também que as maiores influências sobre o modo interárea ocorrem nas linhas 6-7 e 9-8. Isso pode ser explicado pelo fato de essas linhas serem uma extensão da linha de interligação, ou seja, constituem um caminho obrigatório para o fluxo interárea. Porém, observa-se que essas linhas possuem os menores valores de reatância do sistema, limitando desta forma a faixa de compensação e deve-se, portanto, escolher outra linha de transmissão para a inclusão do TCSC (Sadikovic, 2002). Com isto, a linha de interligação entre as barras 7 e 8 torna-se a melhor localização para implementação do TCSC.

O TCSC pode fornecer melhorias consideráveis para a estabilidade dinâmica do sistema, conforme atesta a Tabela 3, que mostra os coeficientes de amortecimento dos modos locais e interárea para as diversas localizações do TCSC. O TCSC realiza uma compensação de $10 \%$ da reatância da linha e seu modelo dinâmico é projetado conforme mostrado na Seção 3.2.

Da Tabela 3 é evidente a relação entre a localização do TCSC 
Tabela 3: Coeficientes de Amortecimento dos Modos Eletromecânicos para diversas Localizações do TCSC.

\begin{tabular}{|c|c|c|c|}
\hline LT & Local 1 & Local 2 & Interárea \\
\hline $1-5$ & 0,5244 & 0,0276 & 0,0343 \\
\hline $2-6$ & 0,5327 & 0,0274 & 0,0241 \\
\hline $5-6$ & 0,5149 & 0,0276 & 0,0340 \\
\hline $6-7$ & 0,0383 & 0,0289 & 0,2430 \\
\hline $7-8$ & 0,0403 & 0,0288 & 0,2049 \\
\hline $9-8$ & 0,0413 & 0,0271 & 0,2272 \\
\hline $3-9$ & 0,0373 & 0,0063 & $-0,0281$ \\
\hline $10-9$ & 0,0375 & 0,5428 & 0,0127 \\
\hline $4-10$ & 0,0374 & 0,5468 & 0,0129 \\
\hline
\end{tabular}

e a inserção de amortecimento para os diferentes modos eletromecânicos de oscilação do sistema.

Analisando os dados da Tabela 3 nota-se que para a localização do TCSC entre as barras 6-7, 9-8 e 7-8, é inserida uma melhora semelhante nos três casos para o amortecimento do modo interárea. Porém, a instalação do TCSC nas linhas 6-7 e 9-8 exige menor esforço do sistema de controle, pois o ganho do controlador se encontra na faixa de 0,05 pu enquanto que para a linha 7-8 este ganho é de 0,4 pu. Este fato pode ser desejado, porém, como já mencionado, as linhas 6-7 e 98 possuem o menor valor de reatância do sistema $(0,01 \mathrm{pu})$, enquanto que a linha entre as barras 7-8 possui reatância de $0,073 \mathrm{pu}$.

Conclusões semelhantes podem ser obtidas com a análise dos fatores de participação do sistema. $\mathrm{O}$ fator de participação $\left(p_{k i}\right)$ mostra a participação relativa da variável de estado $k$ em um determinado modo de oscilação $i$, sendo obtido pela multiplicação entre os autovetores direito e esquerdo, conforme mostra (31) (Kundur, 1994).

$$
p_{k i}=\varphi_{k i} \psi_{i k}
$$

As Figuras 7 e 8 mostram os fatores de participação das variáveis de estado do sistema no modo local 1 e modo interárea, respectivamente. Tais figuras são obtidas com a inclusão do TCSC na linha de interligação entre as barras 7 e 8 .

Analisando as Figuras 7 e 8 nota-se que a reatância do TCSC participa ativamente no modo interárea representado pela Figura 8, proporcionando maior amortecimento para esse modo de oscilação. De acordo com a Tabela 3 e a Figura 7, devido o TCSC estar localizado na linha de interligação, o modo local 1 e local 2 são pouco afetados.

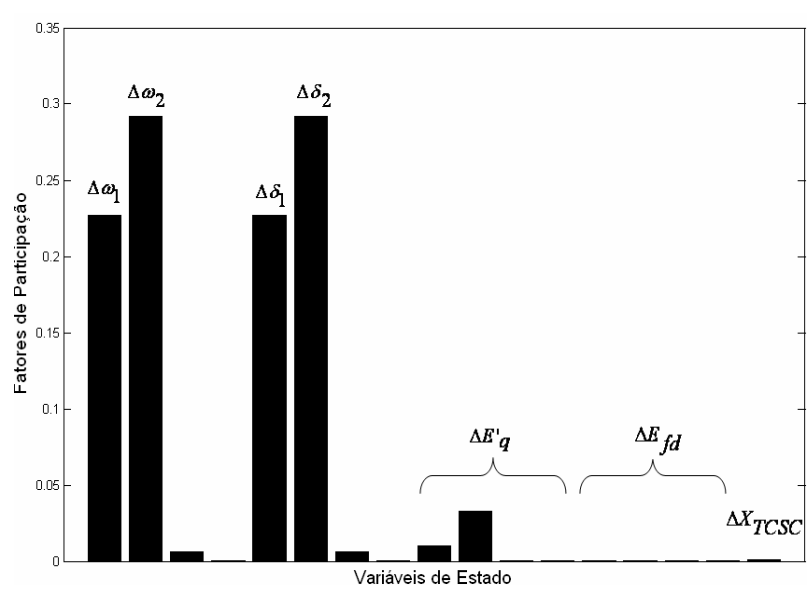

Figura 7: Fatores de Participação para o Modo Local 1

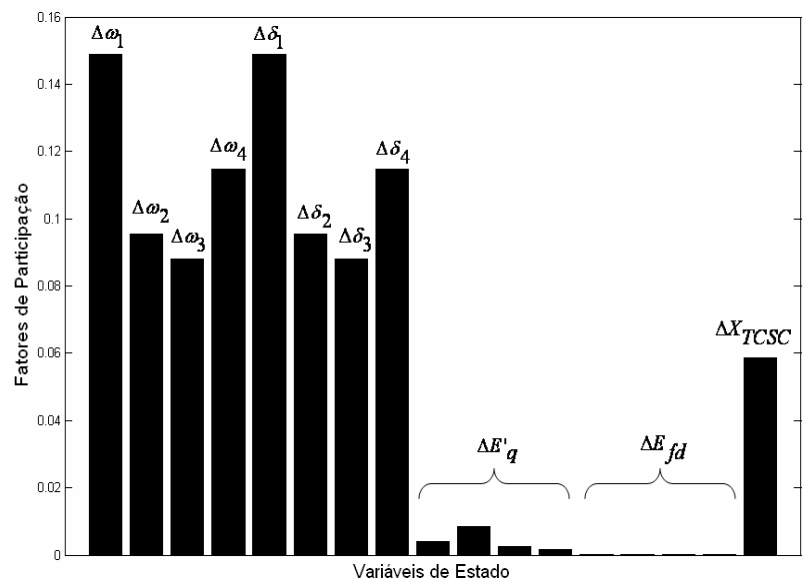

Figura 8: Fatores de Participação para o Modo Interárea

Simulações adicionais mostraram que o aumento indiscriminado de $K_{P O D}$ pode levar o sistema de volta a um ponto de operação instável. Para ganho na ordem de 7 pu, o par de autovalores complexos conjugados associados ao controlador se desloca para o eixo imaginário, tornando-se um par complexo conjugado com parte real positiva. Existe, portanto, uma faixa de ganhos onde se obtém o melhor desempenho do sistema elétrico de potência no que se refere à estabilidade a pequenas perturbações.

\subsection{Efeitos Sobre o Comportamento Di- nâmico do Sistema Elétrico de Potên- cia}

O sistema de energia elétrica é continuamente submetido a pequenas variações de cargas, com os conseqüentes ajustes na geração, através da variação da abertura do dispositivo de captação de água (no caso de usinas hidrelétricas), ocasio- 


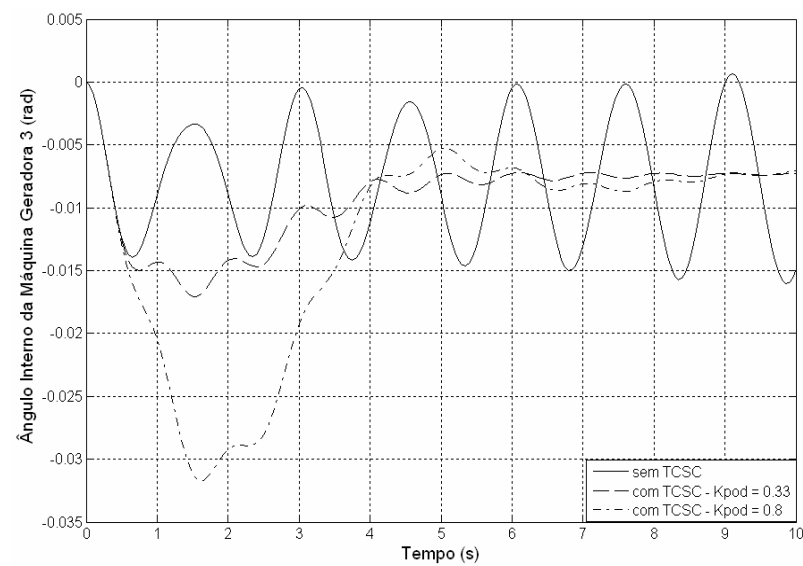

Figura 9: Comportamento Dinâmico do Ângulo Interno do Gerador 3 Devido às Variações do Ganho $\mathrm{K}_{P O D}$

nando um aumento ou diminuição da potência mecânica de entrada dos geradores (Kundur, 1994). Em vista disso, para a análise do desempenho dinâmico do sistema elétrico frente a uma pequena perturbação, considerou-se que para um pequeno aumento na carga do sistema, corresponderá um pequeno ajuste na geração. Este ajuste será aqui representado por um degrau de 0,05 pu na potência mecânica de entrada da unidade geradora 1 .

Na Figura 9 é mostrado o comportamento do ângulo interno da máquina geradora 3 - pertencente à área 2, em relação ao ângulo interno da unidade geradora 1 - pertencente à área 1 , (ou de outra forma, a máquina 1 foi considerada referência para o sistema elétrico), após a ocorrência da perturbação. As diferentes curvas se referem ao caso base, à inclusão do modelo dinâmico do TCSC, cujos parâmetros foram modelados de acordo com a metodologia apresentada na seção 3.2 e apresentados na Tabela 4 e por fim um aumento do ganho estático $\left(K_{P O D}\right)$ do controlador.

Observando a Figura 9 vê-se que o ângulo interno da máquina geradora 3 possui oscilações de amplitude crescente quando o sistema encontra-se sem o controlador TCSC. Porém, quando o controlador é inserido, as oscilações se tornam amortecidas e tendem para um valor final. Ainda de acordo com a Figura 9, o aumento do ganho do TCSC produz excursões maiores nas primeiras oscilações do ângulo interno da máquina geradora 3. Este fato é ocasionado pela diminuição no amortecimento do modo relacionado ao controlador, indicando que, conforme mostrado anteriormente, há uma limitação para o aumento do ganho, fato usual quando se aplica atraso de fase através do controlador POD (Martins et al., 1996). As simulações mostraram as mesmas conclusões para a máquina 4 .

De maneira semelhante ao realizado para a máquina geradora
Tabela 4: Parâmetros do Controlador POD para o TCSC Instalado na Linha de Transmissão entre as Barras 7 e 8.

\begin{tabular}{|c|c|c|c|}
\hline $\mathbf{K}_{P O D}(\mathbf{p u})$ & $\mathbf{T}_{W}(\mathbf{s})$ & $\mathbf{T 1}=\mathbf{T 3}(\mathbf{s})$ & $\mathbf{T 2}=\mathbf{T 4}(\mathbf{s})$ \\
\hline 0.3300 & 1 & 0,0806 & 0,6914 \\
\hline
\end{tabular}

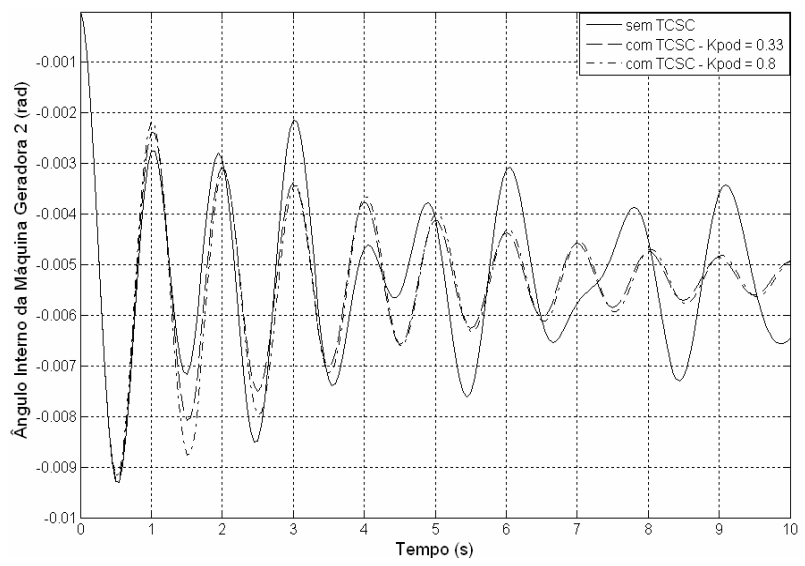

Figura 10: Comportamento Dinâmico do Ângulo Interno do Gerador 2 Devido às Variações do Ganho $\mathrm{K}_{P O D}$

3, foi obtida a Figura 10, referente à máquina geradora 2 (pertencente à área 1).

Analisando-se as curvas da Figura 10, nota-se que a inclusão da função de controle do TCSC leva o sistema a um ponto de estabilidade, porém, aumentos do ganho do controlador não alteram substancialmente o comportamento dinâmico do ângulo interno da máquina geradora 2 . Tal fato pode ser explicado pela localização do TCSC, que atua mais fortemente no modo de oscilação interárea, como pode ser visto a partir dos dados da Tabela 2 (resíduos).

No caso apresentado na Figura 10, a máquina 2 se encontra na mesma área que a máquina 1 , máquina esta que sofre a perturbação. A máquina 2 sente, portanto, os modos locais de oscilação, diferentemente da máquina 3, de área distinta, que sofre influência maior do modo de oscilação interárea.

Conclusões semelhantes podem ser obtidas através da análise do comportamento dinâmico da velocidade angular, conforme mostrado na Figura 11.

Na Figura 11 nota-se claramente a influência do aumento do ganho do controlador nas oscilações da velocidade angular da máquina 3 , em relação à velocidade angular da máquina 1 .

Na Figura 12 têm-se as variações do fluxo de potência ativa nas linhas de transmissão (LT) 7-8, 6-7 e 5-6, devido inclusão 


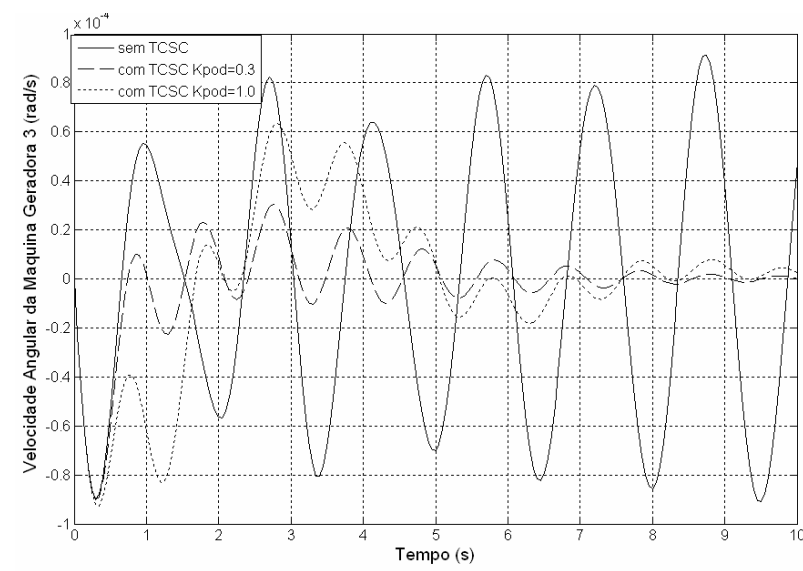

Figura 11: Comportamento Dinâmico da Velocidade Angular da Máquina 3 Devido Variações do Ganho $\mathrm{K}_{P O D}$

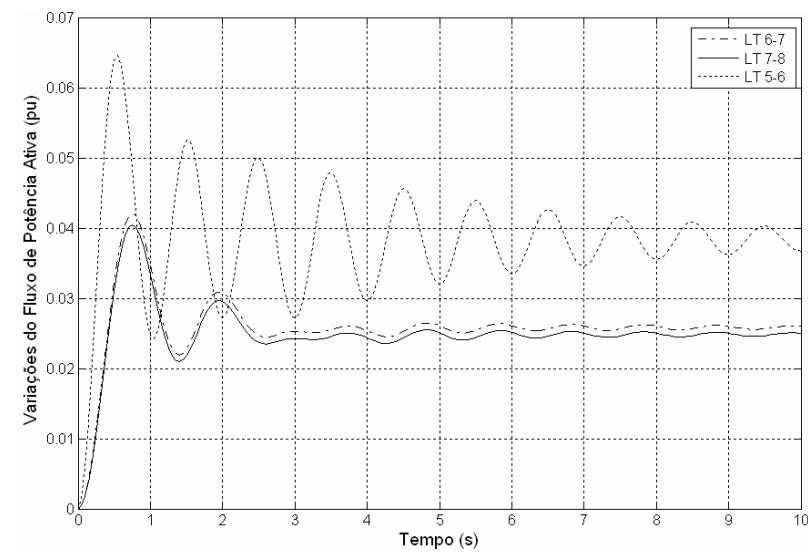

Figura 12: Variações do Fluxo de Potência Ativa nas Linhas de Transmissão entre as Barras 7-8, 6-7 e 5-6.

do TCSC na LT 7-8. Observa-se que o TCSC fornece maior amortecimento para os fluxos de potência ativa nas linhas 7-8 e 6-7. Isto ocorre pelo fato da linha 6-7 ser uma extensão da linha de interligação 7-8, e portanto, ser percorrida obrigatoriamente pelo fluxo de potência interárea conforme descrito anteriormente.

\section{CONCLUSÕES}

Neste trabalho foram apresentados estudos sobre a melhoria da estabilidade de sistemas de energia elétrica a pequenas perturbações, quando da inclusão de um Capacitor Série Controlado a Tiristor. Sabe-se que em grandes sistemas interligados, os modos eletromecânicos de oscilação são geralmente responsáveis pela instabilidade do sistema elétrico. Portanto, quando grandes áreas geradoras são interligadas por linhas longas de alta reatância, pode ser necessária a realização de compensação para se conseguir um ponto de operação estável. Neste trabalho utilizou-se o controlador FACTS TCSC para realizar esta operação.

Da análise realizada através dos autovalores do sistema, concluiu-se que a compensação manual realizada não foi suficiente para levar o sistema à estabilidade. As simulações mostraram que a estabilidade poderia ser obtida se a compensação manual fosse da ordem de $80 \%$ da reatância da linha, o que não corresponde à realidade. Compensações deste porte introduzem outros modos oscilatórios indesejáveis no sistema. Porém, quando um modelo dinâmico para o TCSC foi introduzido notou-se a considerável melhora no amortecimento das oscilações, indicando que foi introduzido amortecimento positivo ao sistema elétrico.

Concluiu-se também que o aumento indiscriminado do ganho do TCSC não garante sempre pontos de operação estáveis, podendo inclusive, tornar o sistema instável.

Outro fato observado foi que a localização do TCSC influencia mais fortemente determinados modos de oscilação. No caso do sistema exemplo, com a instalação na linha de interligação o TCSC introduziu melhorias consideráveis no amortecimento do modo interárea e não dos modos locais. As mesmas conclusões são obtidas pela análise do comportamento dinâmico das variáveis do sistema elétrico.

Do exposto, pode-se concluir que a escolha do valor do ganho e o local da inclusão do controlador TCSC, são de extrema importância para uma compensação que leve o sistema a um ponto estável de operação, pela introdução de amortecimento positivo às oscilações eletromecânicas.

\section{AGRADECIMENTOS}

O presente trabalho foi realizado com ao apoio do Conselho Nacional de Desenvolvimento Cientifico e Tecnológico CNPq - Brasil.

\section{REFERÊNCIAS}

Aboul-Ela M. E., A. A. Sallam, J. D. McCalley and A. A. Fouad (1996). Damping Controller Design for Power System Oscillations Using Global Signals. IEEE Transactions on Power Systems, Vol. 11, No. 2, pp. 767 773.

Anderson P. and A. A. Fouad (2003). Power System Control and Stability. 2th edition, John Wiley \& Sons, INC. Publication, EUA.

Deckmann S. M. and V. F. da Costa (1994). A Power Sensitivity Model for Electromechanical Oscillation Studies. IEEE Transactions on Power Systems, Vol. 9, No. 2, pp. 965-971. 
DeMello F. P. and C. Concordia (1969). Concepts of Synchronous Machine Stability as Affected by Excitation Control. IEEE Transactions on Power Apparatus and Systems, Vol. 88, No. 4, pp. 316-329.

Domingues A. F., J.S. Naturesa, L.C. da Silva, e V.F. da Costa (2002). Aplicação de Dispositivos FACTS para o Amortecimento de Oscilações Eletromecânicas de Baixa freqüência em Sistemas de Energia Elétrica. Anais do XIV Congresso Brasileiro de Automática, Natal - R.N., pp. 1088-1093.

Klein M., G.J. Rogers and P. Kundur (1991). A Fundamental Study of Inter-Area Oscillation in Power Systems. IEEE Transactions on Power Systems, Vol. 6, No. 3, pp. 914-921.

Kundur P. (1994). Power System Stability and Control. Eletrical Power Research Institute - MacGraw-Hill, EUA.

Martins N., Pinto, H. J. C. P., Gama, C., Cavalcanti, J. A., Leoni, R. L.; Souto, R. V., Macedo, N. J. P. and Eiras, M. J. X. (1996). Oscillation Damping Analysis and Control Studies of the Future Interconnection between the North-Northeast and South-Southeast Systems. Proc. of the 5th SEPOPE, Recife - P.E.

Paserba J. J. (2003). How Facts Controller Benefit AC Transmission Systems, Transmission and Distribution Conference and Exposition, IEEE PES, Vol. 3, pp. 991 998.

Pellanda, P. C., Savelli, D. C., Macedo, N. J. P.; Martins, N. e Luz, G. S. (2006). Síntese de Sinais e Escolha de Estrutura dos Estabilizadores dos TCSCs da Interligação Norte-Sul Considerando Robustez a Perturbações Externas. Anais do $10^{\circ}$ SEPOPE, Florianópolis - S.C.

Sadikovic R. (2004). Damping Controller Design for Power System Oscillations. Technical Report, Power Systems Laboratory, Swiss Federal Institute of Technology, Zurick, Switzerland.

Sauer P. W. and M.A. Pai (1998). Power System Dynamics and Stability. Prentice Hall, EUA.

Song Y. H. and A. T. Johns (1999). Flexible AC Transmission System (FACTS). The Institute of Electrical Engineers, United Kingdom.

Yang N., Q. Liu and J. D. McCalley (1998) TCSC Controller Design for Damping Interarea Oscillations. IEEE Transactions on Power Systems, Vol. 13, No. 4, pp. 1304 -1310 .

\section{APÊNDICE A}

Coeficientes do Modelo de Sensibilidade de Potência Multimáquinas.

$$
\begin{gathered}
A 1_{G k}=\frac{E_{q k}^{\prime} V_{k} \cos \left(\delta_{k}-\theta_{k}\right)}{x_{d k}^{\prime}}+ \\
V_{k}^{2}\left[\frac{1}{x_{q k}}-\frac{1}{x_{d k}^{\prime}}\right] \cos 2\left(\delta_{k}-\theta_{k}\right) \\
A 2_{G k}=\frac{V_{k} \operatorname{sen}\left(\delta_{k}-\theta_{k}\right)}{x_{d k}^{\prime}}
\end{gathered}
$$

$$
\begin{aligned}
A 3_{G k}=\frac{E_{q k}^{\prime} \operatorname{sen}\left(\delta_{k}-\theta_{k}\right)}{x_{d k}^{\prime}}+ \\
V_{k}\left[\frac{1}{x_{q k}}-\frac{1}{x_{d k}^{\prime}}\right] \operatorname{sen} 2\left(\delta_{k}-\theta_{k}\right)
\end{aligned}
$$

$$
\begin{gathered}
R 1_{G k}=-\frac{E_{q k}^{\prime} V_{k} \operatorname{sen}\left(\delta_{k}-\theta_{k}\right)}{x_{d k}^{\prime}}- \\
V_{k}^{2}\left[\frac{1}{x_{q k}}-\frac{1}{x_{d k}^{\prime}}\right] \operatorname{sen} 2\left(\delta_{k}-\theta_{k}\right)
\end{gathered}
$$

$$
R 2_{G k}=\frac{V_{k} \cos \left(\delta_{k}-\theta_{k}\right)}{x_{d k}^{\prime}}
$$

$$
\begin{aligned}
& R 3_{G k}= \frac{E_{q k}^{\prime} \cos \left(\delta_{k}-\theta_{k}\right)}{x_{d k}^{\prime}}-\frac{2 V_{k}}{x_{d k}^{\prime}}- \\
& V_{k}\left[\frac{1}{x_{q k}}-\frac{1}{x_{d k}^{\prime}}\right] \cdot\left[1-\cos 2\left(\delta_{k}-\theta_{k}\right)\right]
\end{aligned}
$$

$$
\begin{aligned}
& A 1_{k m}=\frac{R_{k m}}{Z_{k m}^{2}} V_{k} V_{m} \operatorname{sen}\left(\theta_{k}-\theta_{m}\right)+ \\
& \frac{X_{k m}}{Z_{k m}^{2}} V_{k} V_{m} \cos \left(\theta_{k}-\theta_{m}\right)
\end{aligned}
$$

$$
\begin{aligned}
& A 2_{k m}=\frac{R_{k m}}{Z_{k m}^{2}}\left[2 V_{k}-V_{m} \cos \left(\theta_{k}-\theta_{m}\right)\right]+ \\
& \frac{X_{k m}}{Z_{k m}^{2}} V_{m} \operatorname{sen}\left(\theta_{k}-\theta_{m}\right)
\end{aligned}
$$


$A 3_{k m}=-\frac{R_{k m}}{Z_{k m}^{2}} V_{k} \cos \left(\theta_{k}-\theta_{m}\right)+\frac{X_{k m}}{Z_{k m}^{2}} V_{k} \operatorname{sen}\left(\theta_{k}-\theta_{m}\right)$

(A9)

$$
\begin{aligned}
& R 1_{k m}=-\frac{R_{k m}}{Z_{k m}^{2}} V_{k} V_{m} \cos \left(\theta_{k}-\theta_{m}\right)+ \\
& \frac{X_{k m}}{Z_{k m}^{2}} V_{k} V_{m} \operatorname{sen}\left(\theta_{k}-\theta_{m}\right)
\end{aligned}
$$

$R 2_{k m}=-\frac{R_{k m}}{Z_{k m}^{2}} V_{m} \operatorname{sen}\left(\theta_{k}-\theta_{m}\right)+$

$$
\frac{X_{k m}}{Z_{k m}^{2}}\left[2 V_{k}-V_{m} \cos \left(\theta_{k}-\theta_{m}\right)\right]
$$

$$
R 3_{k m}=-\frac{R_{k m}}{Z_{k m}^{2}} V_{k} \operatorname{sen}\left(\theta_{k}-\theta_{m}\right)-\frac{X_{k m}}{Z_{k m}^{2}} V_{k} \cos \left(\theta_{k}-\theta_{m}\right)
$$

\section{APÊNDICE B}

Dados do sistema múltimáquinas utilizado (Sauer e Pai, 1998).

Tabela A1 - Dados das Máquinas Geradoras

\begin{tabular}{|c|c|c|c|c|}
\hline & G1 & G2 & G3 & G4 \\
\hline $\mathrm{x}_{d}^{\prime}(\mathrm{pu})$ & 0,033 & 0,033 & 0,033 & 0,033 \\
\hline $\mathrm{x}_{q}(\mathrm{pu})$ & 0,19 & 0,19 & 0,19 & 0,19 \\
\hline $\mathrm{x}_{d}(\mathrm{pu})$ & 0,2 & 0,2 & 0,2 & 0,2 \\
\hline $\mathrm{H}(\mathrm{s})$ & 54 & 54 & 63 & 63 \\
\hline $\mathrm{D}(\mathrm{pu})$ & 0,1 & 0,1 & 0,1 & 0,1 \\
\hline $\mathrm{T}_{d o}^{\prime}(\mathrm{s})$ & 8 & 8 & 8 & 8 \\
\hline
\end{tabular}

Tabela A2 - Dados do Regulador de Tensão

\begin{tabular}{|c|c|c|c|c|}
\hline Variável & G1 & G2 & G3 & G4 \\
\hline $\mathrm{K}_{r}(\mathrm{pu})$ & 200 & 200 & 200 & 200 \\
\hline $\mathrm{T}_{r}(\mathrm{pu})$ & 0,001 & 0,001 & 0,001 & 0,001 \\
\hline
\end{tabular}

Tabela A3 - Dados das Linhas de Transmissão

\begin{tabular}{|c|c|c|c|c|}
\hline $\begin{array}{c}\text { Barra } \\
\text { Inicial }\end{array}$ & $\begin{array}{c}\text { Barra } \\
\text { Final }\end{array}$ & $\begin{array}{c}\mathbf{R} \\
(\mathbf{p u})\end{array}$ & $\mathbf{X}(\mathbf{p u})$ & $\mathbf{B}(\mathbf{p u})$ \\
\hline 1 & 5 & 0,001 & 0,012 & - \\
\hline 2 & 6 & 0,001 & 0,012 & - \\
\hline 7 & 8 & 0,022 & 0,22 & 0,33 \\
\hline 7 & 8 & 0,022 & 0,22 & 0,33 \\
\hline 7 & 8 & 0,022 & 0,22 & 0,33 \\
\hline 6 & 7 & 0,002 & 0,02 & 0,03 \\
\hline 6 & 7 & 0,002 & 0,02 & 0,03 \\
\hline 4 & 10 & 0,001 & 0,012 & - \\
\hline 3 & 9 & 0,001 & 0,012 & - \\
\hline 9 & 8 & 0,002 & 0,02 & 0,03 \\
\hline 9 & 8 & 0,002 & 0,02 & 0,03 \\
\hline 5 & 6 & 0,005 & 0,05 & 0,075 \\
\hline 5 & 6 & 0,005 & 0,05 & 0,075 \\
\hline 10 & 9 & 0,005 & 0,05 & 0,075 \\
\hline 10 & 9 & 0,005 & 0,05 & 0,075 \\
\hline
\end{tabular}

Tabela A4 - Dados do Ponto de Operação - Caso Base.

\begin{tabular}{|c|c|c|c|c|c|c|}
\hline Barra & $\begin{array}{c}\mathbf{V} \\
(\mathbf{p u})\end{array}$ & $\begin{array}{c}\text { Ang. } \\
\text { (graus) }\end{array}$ & $\begin{array}{c}\mathbf{P}_{G} \\
(\mathbf{M W})\end{array}$ & $\begin{array}{c}\mathbf{Q}_{G} \\
(\mathbf{M V A r})\end{array}$ & $\begin{array}{c}\mathbf{P}_{L} \\
(\mathbf{M W})\end{array}$ & $\begin{array}{c}\mathbf{Q}_{L} \\
(\mathbf{M V A r})\end{array}$ \\
\hline 1 & 1,0 & 8,683 & 700 & 195,97 & - & - \\
\hline 2 & 1,0 & $-2,088$ & 700 & 505,25 & - & - \\
\hline 3 & 1,0 & $-11,92$ & 700 & 601,55 & - & - \\
\hline 4 & 1,0 & 0 & 744 & 236,08 & - & - \\
\hline 5 & 0,97 & 3,846 & - & - & - & - \\
\hline 6 & 0,93 & $-6,928$ & - & - & - & - \\
\hline 7 & 0,88 & $-16,16$ & - & - & 1159 & 212 \\
\hline 8 & 0,86 & $-26,57$ & - & - & 1575 & 288 \\
\hline 9 & 0,92 & $-16,76$ & - & - & - & - \\
\hline 10 & 0,97 & $-5,149$ & - & - & - & - \\
\hline
\end{tabular}

Constante de tempo do Controlador $\mathrm{T}_{T C S C}=0,05(\mathrm{~s})$. 